Musées, Patrimoine et Culture scientifiques et techniques

$156 \mid 2014$

novembre-décembre 2014

\title{
Une expérience singulière : visiter de nuit un musée
}

Floriane Germain

(2) OpenEdition

Journals

Édition électronique

URL : http://journals.openedition.org/ocim/1460

DOI : $10.4000 /$ ocim. 1460

ISSN : 2108-646X

Éditeur

OCIM

Édition imprimée

Date de publication : 1 novembre 2014

Pagination : 17-23

ISSN : 0994-1908

Référence électronique

Floriane Germain, « Une expérience singulière : visiter de nuit un musée », La Lettre de l'OCIM [En ligne], 156 | 2014, mis en ligne le 01 novembre 2016, consulté le 19 avril 2019. URL : http:// journals.openedition.org/ocim/1460 ; DOI : 10.4000/ocim.1460

Ce document a été généré automatiquement le 19 avril 2019

Tous droits réservés 


\title{
Une expérience singulière : visiter de nuit un musée
}

\author{
Floriane Germain
}

La nuit permet des jeux de lumières audacieux comme dans le hall du muséum d'Histoire naturelle de Toulouse.

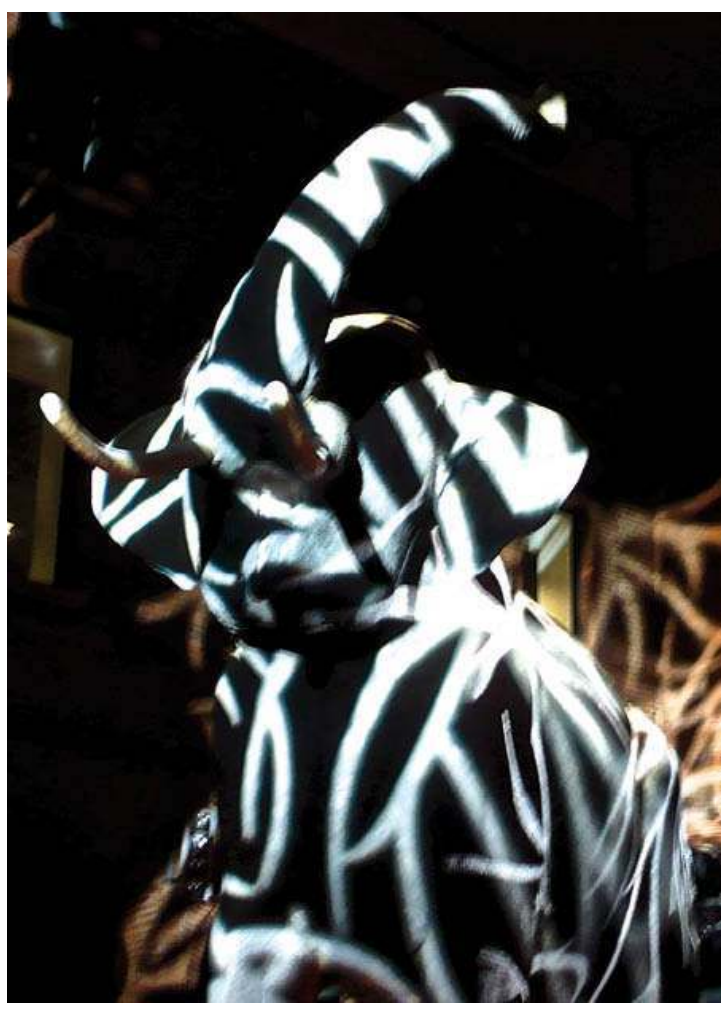

(c) $\mathrm{OCIM} / \mathrm{SL}$

1 L'influence de l'environnement nocturne sur les pratiques de découverte d'une exposition a fait l'objet d'un ensemble de recherches avec un principe simple : comparer 
les pratiques de visite d'une même exposition soit de jour, soit de nuit. Et ce dans le but de mettre en évidence l'influence éventuelle de la nuit et d'une ambiance nocturne sur le processus d'interprétation et de reconnaissance du discours de l'exposition par des visiteurs hommes ou femmes réellement engagés dans cette expérience de visite. Par la comparaison de pratiques de visite diurnes et de pratiques de visite nocturnes, il s'agit de se demander si, pour le public, la variation de temporalité induit (ou n'induit pas) une modification de l'expérience de visite.

\section{L'impact des ouvertures nocturnes sur le choix de venir au musée}

2 Les ouvertures nocturnes font désormais partie de l'offre standard de certains musées, qu'il s'agisse de nocturnes commerciales ou culturelles ou de manifestations événementielles. Les grandes enseignes tout comme les grands musées proposent chaque semaine des ouvertures prolongées qui permettent aux actifs de programmer une activité culturelle après leur journée de travail. À Paris, par exemple, au lieu de rentrer directement chez eux, certains décident de profiter de cette ouverture prolongée pour faire leurs courses ou pour accomplir une activité de loisir culturel, comme la visite de musée. Cette extension des horaires d'ouverture s'inscrit dans un contexte post-moderne que Hartmut Rosa qualifie d'accélération ${ }^{1}$. Changement technique, accélération et multiplication de l'offre génèrent une constante multi-activité et un encombrement de l'agenda qui favorisent le détournement des périodes et du temps autrefois dévolu au repos : la nuit. Quelles sont les particularités de cet espace-temps, et surtout, quel est son impact sur notre perception physique et mentale du monde?

3 La nuit apparaît comme un paramètre ambigu, difficile à cerner ; elle joue à la fois sur la réalité objective puisque c'est une donnée physique naturelle : il fait nuit (pas de lumière naturelle, variation de la température, autre rythme de travail ou social...). Et, en même temps, le changement contextuel entraîne et provoque une perturbation de la perception du monde : la nuit et sa fantasmatique retentissent également sur la subjectivité ; c'est une donnée mentale et psychologique.

4 L'espace-temps de la nuit est toujours défini en opposition à celui du jour et principalement dans ses représentations. Le jour est la période associée à la lumière, au savoir, à la raison triomphante, toutes des notions positives. La nuit en revanche est associée aux ténèbres et à l'ignorance, au délit et à la transgression, toutes des notions négatives. Cette opposition tranchée se retrouve de manière plus nuancée dans la réalité. Longtemps la nuit a été une période de repli dans l'intimité du foyer, auprès de la source lumineuse, réconfortante non seulement par sa chaleur mais aussi par sa lumière qui chasse les ténèbres environnantes. Ce sont ces lumières qui permettent à l'Homme d'habiter la nuit, d'y avoir des activités. Sans elles, on ne peut plus se fier au sens de la vue, les autres sens doivent pallier cette défaillance, notamment l'ouïe, et nous apportent alors une appréhension différente de notre environnement. Cette appréhension est liée à la peur du noir qui cache des dangers imaginaires et réels. Toutes sortes de représentations investissent notre imagination, un porte-manteau peut alors se transformer en monstre.

5 La nuit est donc un stimulus qui agit sur les sens (par les perceptions) mais aussi sur les représentations (à la fois sociales et mentales). Les visites nocturnes se nourrissent de ces 
représentations issues de la nuit. Dans la culture populaire, de nombreuses œuvres de fiction reprennent le temps de la visite de nuit sous une forme fantasmée. La nuit, des choses mystérieuses se déroulent au musée: meurtre, énigme, fantômes ou encore œuvres revenant à la vie. Parmi les plus célèbres de ces œuvres on trouve le Da Vinci Code (2004), Belphégor (1965) ou encore La nuit au musée (2008). Qu'en est-il dans la réalité ? Le déplacement de la pratique de visite dans la nuit peut-il à lui seul entraîner une différence dans la pratique ou la perception de cette activité ?

L'image mystérieuse de la visite nocturne doit pouvoir influencer les visiteurs et les théories de la réception - et notamment de la réception esthétique de Jauss - rendent plausible ce changement d'interprétation de l'exposition ${ }^{2}$. En effet, pour lui le potentiel de signification de l'œuvre change selon le contexte mais aussi selon les caractéristiques sociodémographiques du récepteur et ses motifs de visite. Le changement contextuel jour/nuit pourrait ainsi entraîner en lui-même des variations notamment par les modifications d'univers de représentations et de perceptions ; la nuit pourrait aussi, parce qu'elle ne concerne qu'un public spécifique, sélectionner de facto certains horizons d'attente ou motifs de visite chez les visiteurs. Les recherches sur l'expérience de visite et la réception de l'exposition ont tenté d'échapper au déterminisme sociologique dénoncé en son temps par Pierre Bourdieu. Elles ont mis en évidence des rapports plus nuancés et surtout plus hétérogènes entre l'offre d'exposition et les motivations et attentes des visiteurs. On peut avancer que l'expérience de visite est fortement influencée par l'état d'esprit des visiteurs au moment de la visite et par le contexte de cette visite.

Au planétarium de Strasbourg, les ombres projetées modifient la perception des objets exposés.

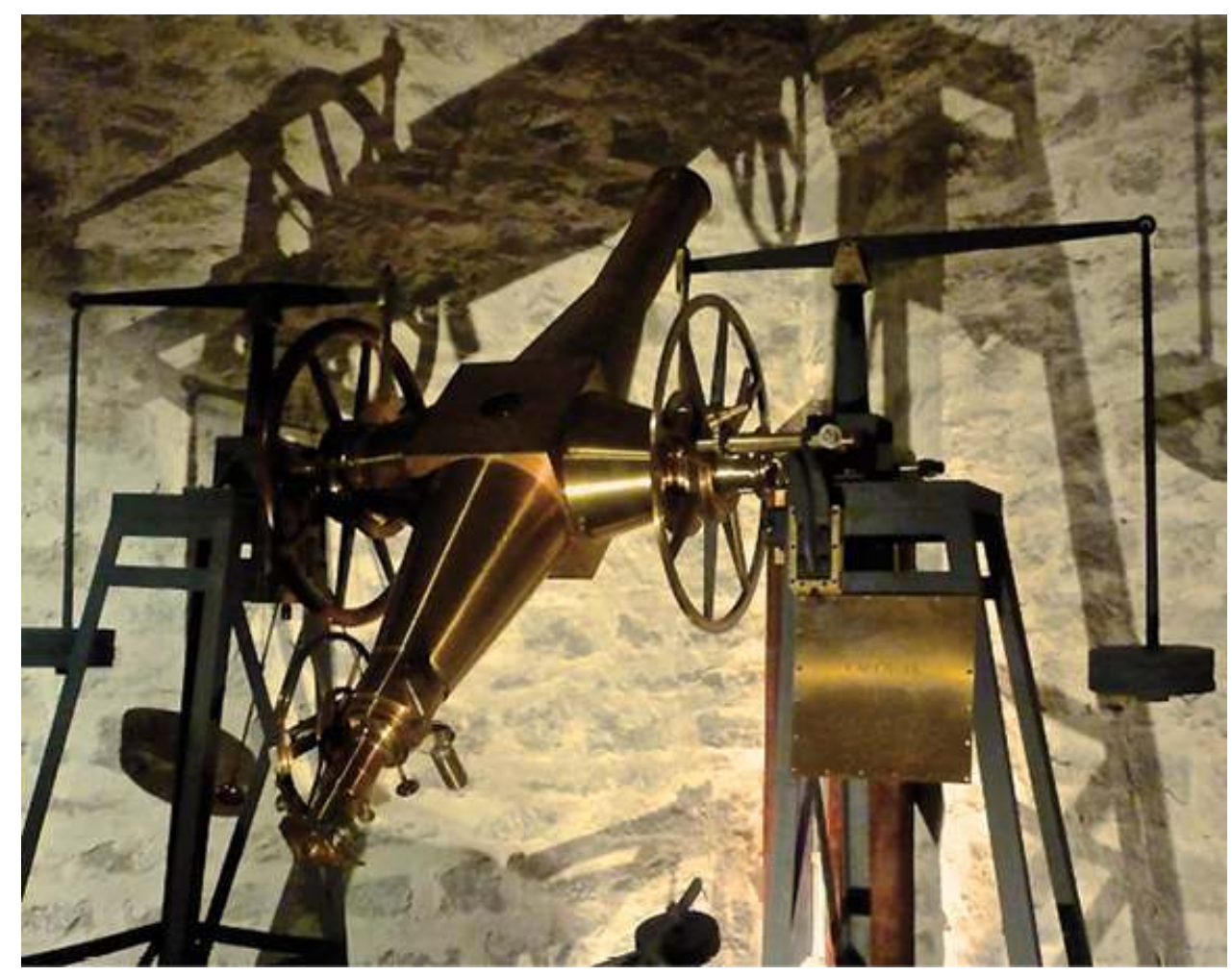

(c) $\mathrm{CIM} / \mathrm{SL}$

6 Chaque changement dans le contexte de l'exposition mais également chaque changement dans l'état d'esprit des visiteurs peuvent donc potentiellement générer une expérience de 
visite différente (Falk, 2009). Étant donné le rôle majeur du contexte dans cette expérience, il est donc presque évident que le fait de visiter un musée la nuit au lieu du jour apporte un changement dans cette expérience de visite. De plus, les motifs de visite pourraient varier entre jour et nuit si les visiteurs s'attendent à vivre une expérience différente de celle du jour.

\section{Les particularités de la nuit et leurs conséquences sur l'étude de réception}

7 Une étude de réception d'une exposition de nuit doit prendre en compte le changement d'espace-temps que représente la nuit pour accéder à l'interprétation de son message par les visiteurs. Selon la conception de l'exposition comme un média dont le fonctionnement s'appuie sur l'interaction objet/visiteur, la création du sens dans l'exposition est personnelle. Elle se fonde sur la subjectivité des visiteurs et leur parcours de visite mais également sur l'agencement spatial de l'exposition et de ses registres. Le visiteur est actif au cours de sa visite d'exposition, il fait des choix sur ce qu'il regarde, sur ce qu'il lit, sur son itinéraire. Selon son intérêt, il pioche des morceaux du texte composite de l'exposition, à travers les objets, la mise en espace et les textes. À partir de ces bribes de discours d'exposition, il compose ensuite sa propre compréhension du texte d'exposition et donne son propre sens à l'exposition. Ainsi, en parcourant ce texte ouvert qu'est l'exposition, comme Umberto Éco l'avait dit du livre, les visiteurs construisent leurs propres scénarios de l'exposition. Ils participent de la création finale du sens donné à ce qu'ils rencontrent. Dans ce cas, le changement de contexte de la visite (passage du jour à la nuit) et plus particulièrement le changement de luminosité (un degré de lumière mais aussi une qualité différente) mettrait en jeu d'autres sensations, qui entraîneraient d'autres rapports aux objets, dont découleraient d'autres interprétations et, finalement, un sens différent donné à la visite. Le tout selon une chaîne de création du sens induite par des sensations.

Cette configuration de coopération interprétative suppose que la subjectivité et le contexte entrent en jeu dans la création du sens. Or, l'étude de ce changement dans l'expérience de l'exposition de jour et de nuit, est aussi suggérée par les théories sémiotiques, plus particulièrement celle de Peirce. Selon lui tout ce qui nous entoure est potentiellement un signe porteur de sens et interprétable. Tout dans l'exposition peut donc être pris en compte (le visiteur lui-même mais aussi les autres visiteurs, le discours, les objets ou les œuvres, l'espace et le contexte) en tant que signe engageant un processus d'interprétation éminemment subjectif. Cette subjectivité se lie à l'expérience de la personne mais aussi au contexte dans lequel se déroule l'interprétation. 
La cour de Marly au Louvre, la nuit.

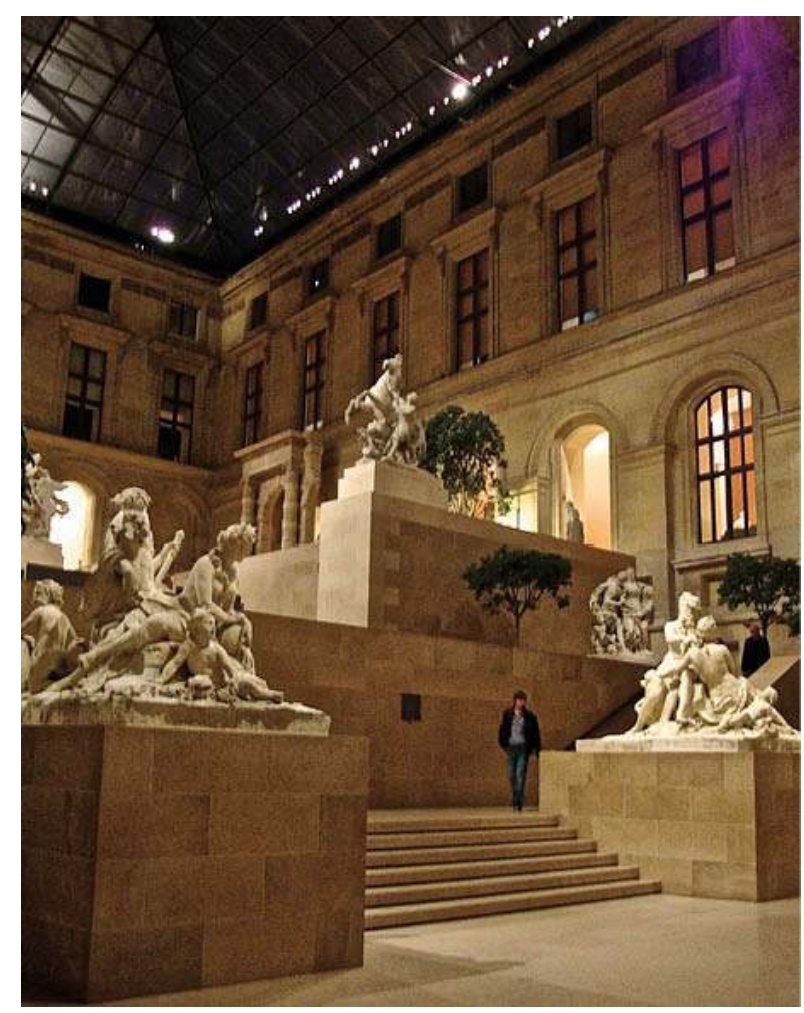

(C) F. Germain

Cet impact de la nuit sur la réception d'une exposition et la variation de sens qu'elle peut entraîner dans l'interprétation des visiteurs ont fait l'objet d'une enquête au musée du Louvre de décembre 2011 à février 2012. Elle s'est plus particulièrement déployée dans la cour Marly de l'aile Richelieu au rez-de-chaussée du musée, qui rassemble les sculptures françaises des XVIIe-XVIIIe siècles. Elle abrite la grande sculpture exécutée pour le parc du château de Marly. L'architecte de la pyramide du Louvre, Ieoh Ming Pei, a recouvert la cour d'une verrière en 1993, lors des travaux du Grand Louvre, ce qui donne un attrait particulier à ce vaste espace et laisse y pénétrer sans entrave la lumière naturelle. Par cette ouverture plein ciel sur l'extérieur, la cour Marly du Louvre est particulièrement indiquée pour examiner les variations d'ambiance et de contexte entre jour et nuit. L'intérêt majeur de cette cour a été de permettre d'interroger véritablement l'impact de la nuit sur la visite de ce fragment d'exposition permanente du musée.

10 Une analyse experte de la cour révèle une muséographie marquée avec un espace fortement scénographié qui cherche à recontextualiser les œuvres. En effet, la cour est composée de terrasses qui modélisent chacune une partie du parc en recréant des groupes de sculptures provenant des mêmes bosquets. Les ensembles stylistiques et iconographiques du parc sont ainsi conservés.

11 Entre le passage du jour à la nuit, seul l'éclairage subit une variation, passant d'un éclairage naturel à un éclairage artificiel. Une série d'entretiens a été menée dans la cour Marly, de jour et de nuit, auprès de 126 visiteurs francophones se répartissant équitablement entre jour et nuit, 63 entretiens pour chaque groupe. De plus, 200 personnes ont été observées, 100 observations de jour et encore 100 pendant les nocturnes. 
12 Les entretiens menés au cœur de la cour Marly couplés à l'observation des visiteurs mettent en évidence le poids de la nuit et de son cortège de représentations sur les perceptions et l'interprétation et au-delà sur la nature de l'expérience de visite.

\section{La variation du sens donné à la visite et la singularité de la visite de nuit}

13 Les résultats de l'enquête montrent que des différences existent bel et bien entre jour et nuit dans l'interprétation et la réception de l'exposition. Il apparaît que la variation du sens est faite d'un ensemble de petites modifications: différence de perception, différence de vision des œuvres, différence de ressenti, différence d'ambiance, différence d'état d'esprit, différence de rythme, différence d'agenda personnel et en définitive différence de public.

Ces variations sont principalement basées sur le ressenti de l'ambiance et sur la perception des autres visiteurs. En ce qui concerne l'impact de l'ambiance, il est accessible à travers l'interprétation sensorielle que les visiteurs donnent à leurs visites. Elle est soutenue par les conditions de visite, mais aussi par la scénographie et l'éclairage. La première donnée de cette étude sur l'ambiance des visites nocturnes est que les visiteurs de nuit semblent avoir une approche plus sensible de l'exposition à travers une interprétation fondée davantage sur les émotions et l'imagination que sur le cognitif. Là où les visiteurs du jour montrent une plus grande volonté de compréhension des œuvres et de l'exposition. Ainsi, la journée les visiteurs chercheront des informations sur les œuvres alors que la nuit ils s'intéresseront plus à leur côté esthétique. L'objectif de la visite de nuit semble alors être moins axé sur l'apport de connaissances et plus sur l'émotion et la décontraction. La convivialité et l'expérience sociale du musée sont une des expressions majeures de la visite de nuit. Les observations réalisées au musée du Louvre, montrent d'ailleurs qu'une différence importante apparaît entre les visiteurs de jour et les visiteurs de nuit concernant le traitement des textes. La journée, les visiteurs cherchent des informations sur les panneaux et sur les cartels, la nuit quelques visiteurs lisent des cartels mais l'activité globale de lecture observée est largement inférieure à celle de la journée. En revanche, les visiteurs de nuit font beaucoup plus le tour des œuvres. Si les visiteurs ne lisent pas les textes dans la cour Marly, il est légitime de conclure qu'ils ne cherchent peut-être pas à s'orienter conceptuellement dans la cour. La traversée de la cour se baserait plutôt sur une relation sensorielle à leur environnement marquée par quelques points d'intérêts visuels et esthétiques majeurs. Ainsi, les visiteurs de nocturnes feront plus d'arrêts dans la cour, plus longs qu'en journée, et prendront aussi plus de photographies, ils regarderont plus souvent autour d'eux ou en l'air et interagiront plus avec leurs compagnons. D'ailleurs, le rythme de la visite est une préoccupation plus forte chez les visiteurs de nocturnes, qui semble traduire une recherche de conditions de visite spécifiques permettant une certaine expérience de visite, plutôt liée à la détente. La visite nocturne semble donc être une visite libre, sans souci d'apprentissage formel, une activité sociale qui permet de se détendre et de bavarder avec en toile de fond les œuvres d'art. D'ailleurs, la visite nocturne permet une coupure dans la journée voire la semaine de travail et permet aux personnes interrogées de se changer les idées, de se ressourcer. 
La différence de perception des sculptures de nuit et de jour : La Renommée sur Pégase (1699-1702) dans la cour Marly du Louvre.

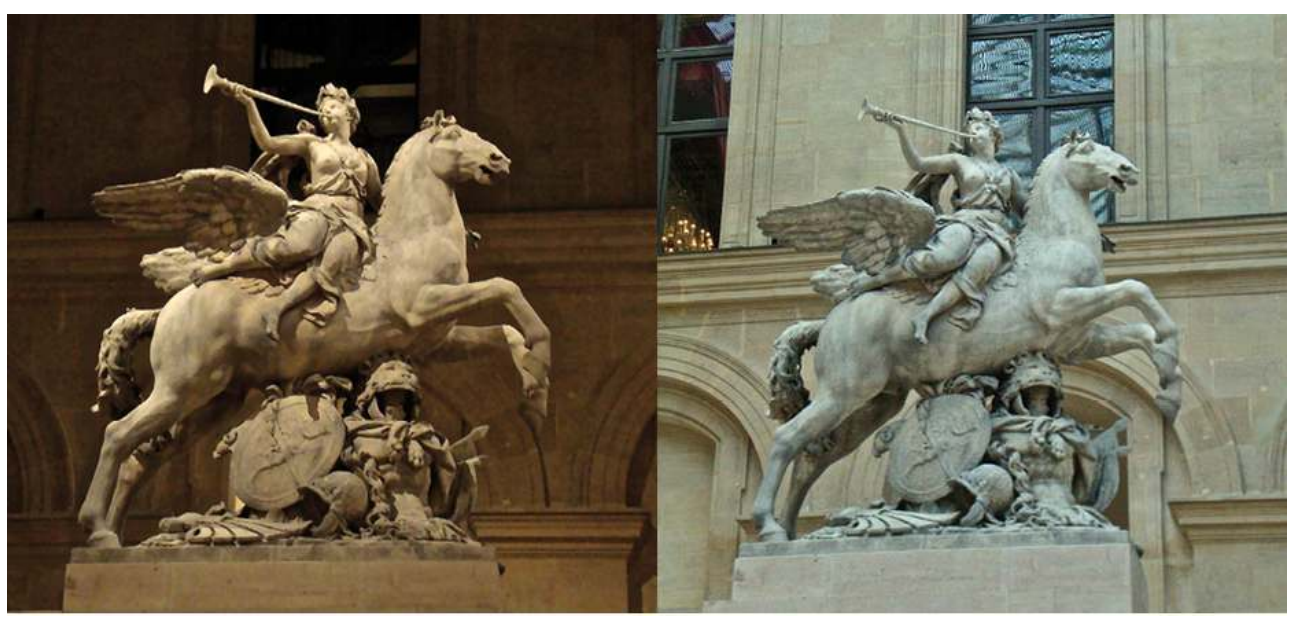

(c) F. Germain

15 Dans ce changement de perception du lieu, l'éclairage joue un rôle clé. En effet, la différence majeure entre une visite de jour et une visite de nuit est le passage d'un éclairage naturel à un éclairage artificiel dirigé. Le changement d'éclairage entre jour et nuit entraîne une perception différente du lieu et des œuvres. Ainsi, l'éclairage artificiel de nuit entraîne une perception plus dramatique des œuvres qui leur donne un côté plus vivant, voire impressionnant. Par le jeu des ombres et de la lumière, l'éclairage nocturne recompose la perception des volumes de l'œuvre et génère une ambiance générale qualifiée de plus intime.

16 L'étude sensible de la visite nocturne montre donc un certain glissement des motifs de visite et des sensations expérimentées au cours de la visite. Cet état de fait est lié à l'état d'esprit des visiteurs de nuit qui affirment rechercher une approche plus imaginative de la visite. Les témoignages recueillis de nuit contiennent notamment plus de références au voyage dans le temps, à l'aspect vivant des œuvres et à la sensation de vivre une aventure. La nuit devient un cadre d'interprétation des visiteurs divergent notamment à cause de sa dimension fantasmatique.

Il est également apparu lors de cette enquête que les visiteurs de nocturnes avaient un retour critique sur leur pratique de visite et par là même sur celle des autres visiteurs. Les visiteurs de nuit se ressentent comme différents de ceux de jour. Il existe en effet des différences de profil chez les visiteurs de nocturnes. Tout d'abord, les écarts habituellement repérés entre la présence d'hommes et de femmes se réduisent la nuit. Les femmes sont toujours les plus présentes au musée mais le nombre d'hommes présents augmente. De même, proportionnellement les 20-24 ans, 35-44 ans et 45-54 ans sont plus présents en nocturne qu'en journée. En ce qui concerne la compagnie des visiteurs, les publics de la nuit au musée sont plutôt des adultes venus entre amis ou en couple. Les amis sont plus souvent interrogés en nocturne. La visite nocturne pourrait donc être une visite entre amis plus que la visite de jour qui serait peut-être plus familiale. Les visiteurs de nocturnes sont principalement des actifs hauts diplômés et semblent généralement d'origine parisienne tandis que les visiteurs du jour seraient plus globalement des touristes, nationaux ou internationaux et en ceci, caractéristiques du public du musée du Louvre. Il y aurait donc un changement de populations entre jour et nuit, qui est en effet 
ressenti par les visiteurs, et qui pourrait s'expliquer par l'horaire allongé qui permet à des personnes actives la journée de se rendre au musée pour y passer la soirée, comme ils iraient au cinéma après leur journée de travail. Les visiteurs de nuit sont aussi des visiteurs plus fréquents des musées et ont une plus grande habitude muséale, il y aurait a priori une plus grande représentation de visiteurs assidus chez les personnes interrogées de nuit.

Le profil des visiteurs des nocturnes correspond ainsi globalement à l'image que s'en font les visiteurs interrogés de nuit. Pour eux, la personne qui profite des nocturnes pour visiter le musée est avant tout un connaisseur et un amateur. À l'inverse, pour eux, les visiteurs de jour sont avant tout des touristes, une différence qu'ils acceptent (ou recherchent?) la foule et le bruit. Ainsi, venir en nocturne permet d'éviter ces groupes de touristes bruyants et transforme les conditions de visite. D'agressif et fatigant, l'environnement muséal devient paisible et reposant et incite les visiteurs à prendre leurs aises, physiquement et psychologiquement. Les visiteurs de la nuit cherchent donc à se distinguer des visiteurs de jour dans leur attitude, leurs habitudes des musées et leur représentation exigeante de la visite.

La visite nocturne au musée : une visite particulière.

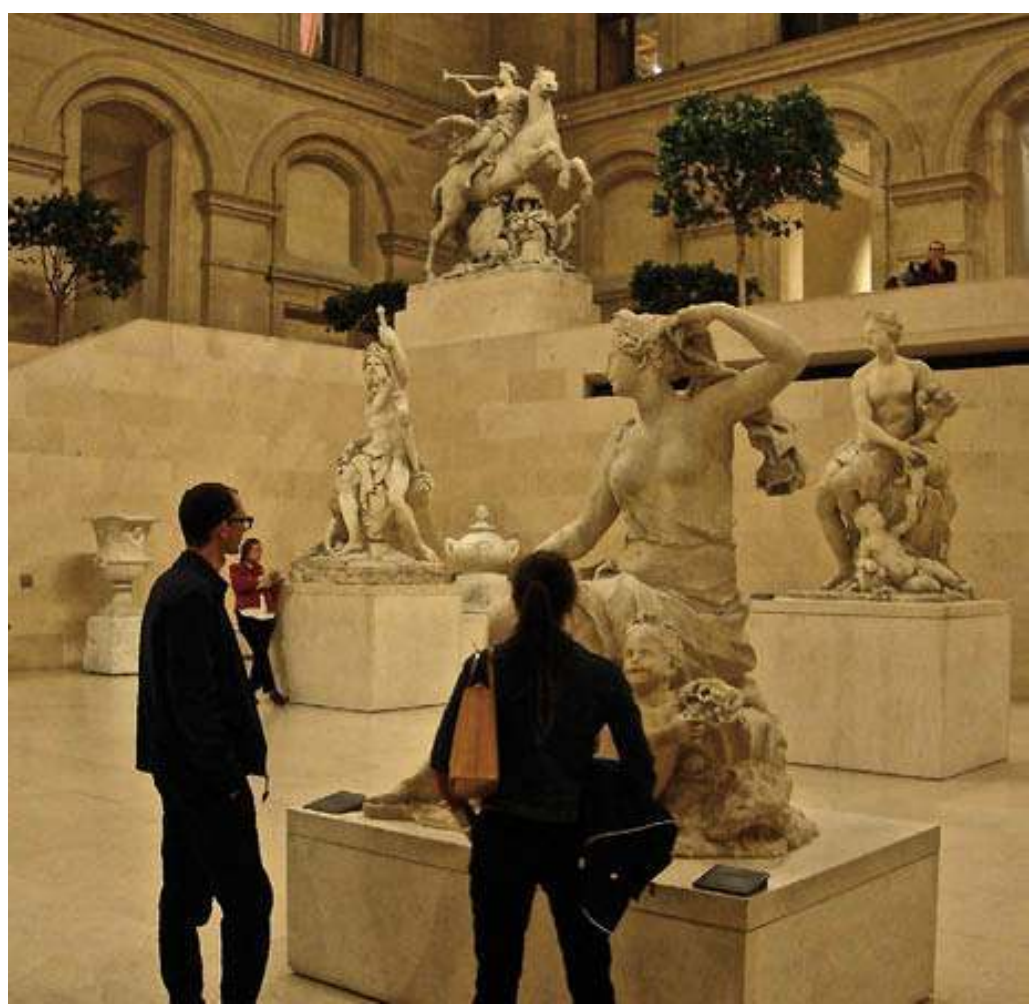

(c) F. Germain

En suivant la méthodologie d'analyse du sens développée par Peirce ${ }^{3}$, il apparait que le développement de la visite du musée pendant la nuit est rendue possible par le point de vue associé à cette visite, c'est-à-dire la fantasmatique de la nuit qui est liée à un état de perception du monde différent (Peirce, 1998). C'est cet état qui détermine la visite de musée la nuit en tant que comportement à travers le parcours de l'exposition et la vision des œuvres. Visiter la nuit est alors interprété en fonction du décalage créé avec une visite de jour qui entraîne une certaine délectation des visiteurs qui constitue à son tour 
une visite singulière se distinguant des autres. Ceci va finalement pouvoir entraîner une vision différente de l'exposition voire de la visite de musée en général.

Cette variation sémiotique, fréquemment interrogée, reflète la subjectivité de la production du sens. À l'échelle d'une exposition, la variation se produit dans l'interprétation mais aussi dans le contexte de visite et se traduit par le repérage des éléments ne changeant pas d'un contexte à l'autre face à ceux qui montrent des différences (Peytard, 1993).

La nuit au musée d'Art moderne et d'Art contemporain de Nice : des perspectives inhabituelles et des angles de vue particuliers.

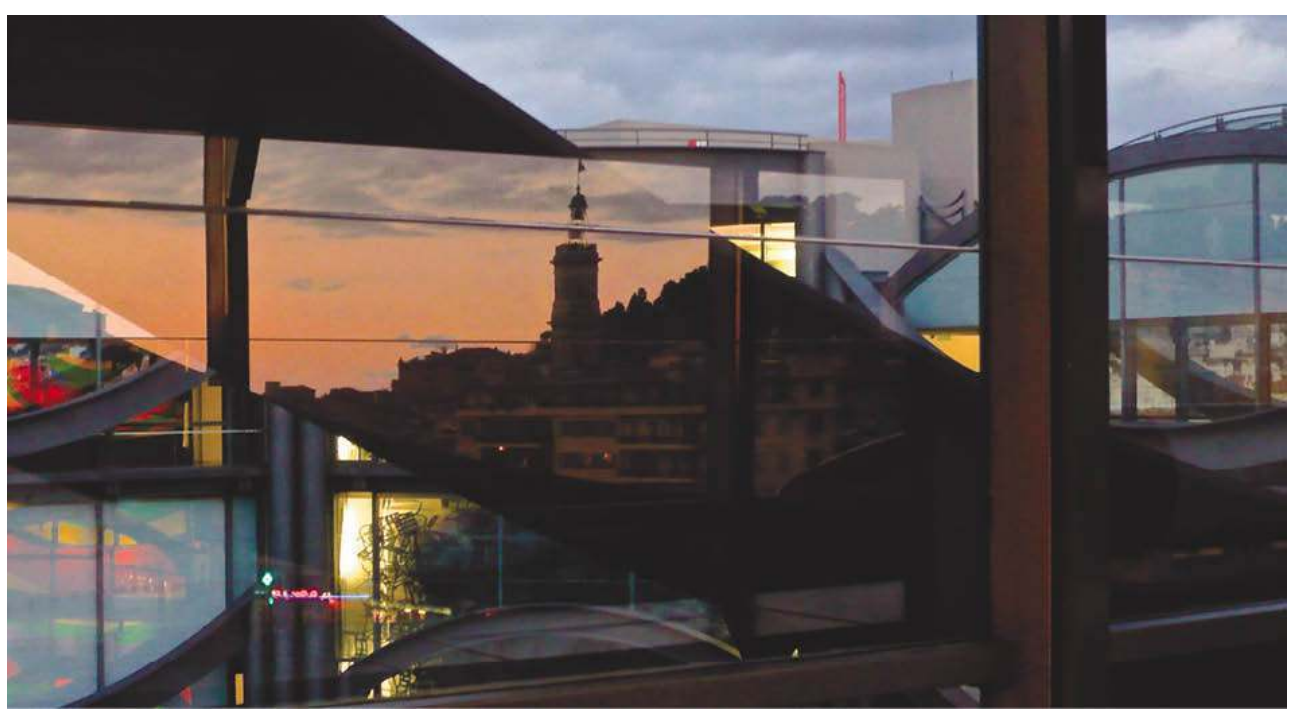

(c) $\mathrm{OCIM} / \mathrm{SL}$

21 La comparaison musée de nuit/musée de jour pose la question du même. Cette variation fait-elle déjà du même un autre ? C'est ce que le sémioticien Jean Peytard avait nommé la sémiotique de l'altérité. Quelle est la plus petite variation qui à elle seule peut modifier le sens? Avec l'arrivée de la nuit, le musée semble passer de lieu ouvert au plus grand nombre, à un palais ouvert à quelques privilégiés. Ce changement est ressenti à travers le changement lumineux qui soutient le côté mystérieux et intime du lieu accompagné de toute sa fantasmatique, mais aussi à travers les changements de conditions de visite. Le fait qu'il y ait moins de monde et que les visiteurs se sentent entourés d'autres personnes appréciant le musée comme eux, leur accorde une plus grande liberté dans leurs manières et dans leur maintien. Finalement, ils engagent un rapport différent au musée en tant qu'activité sociale de loisir.

Il s'avère compliqué de départager toutes ces composantes de l'expérience de visite pour mesurer le poids de chacune d'elles dans la variation du sens. Au sein de la cour Marly, le lieu en lui-même ne subit pas de variation autre que celle du changement de lumière. Les œuvres et les aides à la visite sont toujours les mêmes, la scénographie aussi. Le changement d'impact du lieu ne se trouve d'ailleurs pas dans l'impression de la pièce puisque, de jour et de nuit, les visiteurs s'accordent sur la monumentalité de la cour et son calme qui en font un endroit reposant et agréable qui permet de faire une pause dans sa visite des salles du musée, comme un jardin. Le changement de comportement et de perception serait donc déclenché par la présence de la nuit qui, plutôt que de recomposer du tout-au-tout une ambiance dans la cour, influence l'état nues en nocturne. Non 
seulement la présentation des œuvres est légèrement différente par l'abaissement de la luminosité, qui devient plus jaune, mais encore les visiteurs recherchent cette variation qui fait de la nocturne une visite singulière. C'est venir visiter de nuit qui permet d'expérimenter une nouvelle manière de visiter, dans des conditions qui sont vues comme privilégiées.

Il existe donc une variation du sens donné à la visite selon qu'elle se déroule de jour ou de nuit. Cette variation est avant tout supportée par l'aspect subjectif et sensible de l'expérience de visite notamment par le biais des représentations de la visite de nuit et plus largement par l'imaginaire de la nuit.

Les publics du jour et de la nuit ont une démarche et des états d'esprit opposés qui sont largement tributaires du contexte diurne ou nocturne de leur venue. Ils vont alors expérimenter une visite différente qui est liée, ou influencée, par la manière dont ils définissent leur rôle de visiteur de musée et partant par la manière dont ils définissent le musée, outil et lieu d'épanouissement de leur image de visiteur. L'interprétation du message de l'exposition est alors spécifique : elle coïncide avec le type d'expérience de visite que les visiteurs de nuit recherchent et que les visiteurs de jour subissent. Par cette reformulation (cour le jour, cour la nuit), l'état originel du discours de la cour Marly n'est plus le même. Il est altéré à la fois par une autre interprétation des œuvres et une autre représentation sociale de la figure de visiteur qui résultent elles-mêmes d'une autre pratique du musée que les visiteurs ont délibérément choisie.

\section{BIBLIOGRAPHIE}

Brown, D. Da Vinci Code. Paris : Le Grand Livre du mois, 2004, 571 p.

Cabantous, A. Histoire de la nuit, XVIIe-XVIIIe siècle. Paris : Librairie Arthème Fayard, 2009, $387 \mathrm{p}$.

Davallon, J. L'exposition à l'œuvre : stratégies de communication et médiation symbolique. Paris : L'Harmattan, 2000, $384 \mathrm{p}$.

Dorgelès, R. Le Palais réveillé. Vie Parisienne, 25 mai 1918. Paris, 1918.

Ebrard, G. (dir.) Tourisme et lumière : Guide pratique de l'animation nocturne des villes, sites et monuments. Rapport pour la Section de l'aménagement touristique, Conseil national $\mathrm{du}$ tourisme. Paris : La Documentation française, 1998.

Éco, U. Lector in fabula ou la Coopération interprétative dans les textes narratifs. Paris : Grasset, 1985, 320 p.

Falk, J.-H. Identity and the museum visitor experience. Walnut Creek, États-Unis : Left Coast Press, 2009, $301 \mathrm{p}$.

Guérin, C. Nocturnes gratuites en octobre au musée du Louvre pour les étudiants, Publics et Musées, n 5, 1994, pp. 122-125.

Jauss, H.R. Pour une esthétique de la réception. Paris : Gallimard, 1978. 
Peirce, C.-S. \& Burks, A.-W. Collected papers of Charles Sanders Peirce. 8, Reviews, Correspondence, and Bibliography. Bristol, Royaume-Uni : Thoemmes Press, 1998.

Peytard, J. D’une sémiotique de l'altération, Semen, nº 8, 1993. Repéré sur http:// semen.revues.org/4182

Rosa, H. Accélération : une critique sociale du temps. Paris : La Découverte, 2010, 475 p.

Roustan, M. Les soirées exceptionnelles du Louvre. Publics, logiques d'usage, modes de réception. Paris : Musée du Louvre, 2011.

Verlet, P., Vandier, J., Aubert, M., Parrot, A., Devambez, P. et Albin-Guillot, L. Au Louvre la nuit, texte. Paris : Arthaud, 1952.

Verne, H. Le Louvre la nuit. Grenoble : Arthaud, 1937, 115 p.

\section{NOTES}

1. Hartmut Rosa est sociologue et philosophe, auteur de l'ouvrage Accélération : une critique sociale du temps. Paris : La Découverte, 2010, 475 p.

2. Hans Robert Jauss (1921-1997) est un philosophe et théoricien allemand de la littérature connu pour sa théorie de la réception, auteur de l'ouvrage Pour une esthétique de la réception. Paris : Gallimard, 1978.

3. Charles-Sanders Peirce (1839-1914) est un sémiologue et philosophe américain.

\section{RÉSUMÉS}

Une enquête réalisée auprès des visiteurs nocturnes du musée du Louvre a permis de mettre en évidence les particularités de cet espace-temps, de comprendre pourquoi et comment la nuit agit sur les perceptions et les représentations de l'exposition et plus généralement de l'espace muséographique par les visiteurs, modifiant ainsi leur expérience de visite.

\section{INDEX}

Mots-clés : visite, nuit, musée, nocturne

\section{AUTEUR}

\section{FLORIANE GERMAIN}

Doctorante du programme international muséologie, médiation, patrimoine, programme conjoint entre l'université d'Avignon et des Pays de Vaucluse, l'université du Québec à Montréal et l'École du Louvre à Paris

floriane.germain@yahoo.fr 